\title{
Erratum: Stellar properties and nuclear matter constraints [Phys.Rev.C93,025806(2016)]
}

\author{
Mariana Dutra \\ Departamento de Ciencias da Natureza - IHS, Universidade Federal Fluminense, 28895-532 Rio das Ostras, RJ, Brazil \\ Odilon Lourenço \\ Universidade Federal do Rio de Janeiro, 27930-560, Macaé, RJ, Brazil
}

Débora P. Menezes

Departamento de Física - CFM - Universidade Federal de Santa Catarina, Florianópolis - SC - CP. 476 - CEP 88.040 - 900 - Brazil

email: debora.p.m@ufsc.br

(Dated: September 16, 2021)

\begin{abstract}
In this paper we show the corrected results related to two density dependent relativistic mean field parametrizations analyzed in a previous work and review some of our conclusions, mainly the one that three models produce stars with maximum masses above recent observational results, whilst in fact, only two of them can do so.

PACS numbers: 21.30.Fe, 21.65.Cd, 26.60.Kp, 24.10.Jv
\end{abstract}

In Ref. [1], we analyzed stellar properties of the relativistic mean-field (RMF) parametrizations shown to be consistent with nuclear matter, pure neutron matter and symmetry energy constraints. Among our conclusions, we stated that three models yield neutron stars with maximum masses above that pointed out by recent observational results of about $2 M_{\odot}[2,3]$. Unfortunately, there was a technical mistake in the calculation of maximum stellar masses of two of the parametrizations with density dependent couplings and $\delta$ mesons, namely, DDH $\delta$ and DD-ME $\delta$, which resulted in wrong conclusions. Corrected tables and figures are presented here. In corrected Table I, we show only the lines related to these two parametrizations.

TABLE I. Corrected results of DDH $\delta$ and DD-ME $\delta$ for neutron star main properties: maximum stellar masses in terms of $M_{\odot}$

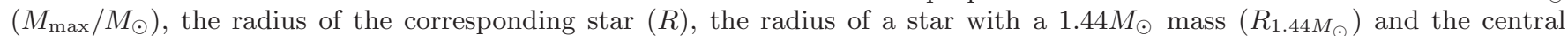
energy density of the maximum mass star $\left(\varepsilon_{c}\right)$. Properties related to the direct Urca process: mass of the star in terms of $M_{\odot}$ at the onset of the DU process $\left(M_{\mathrm{DU}} / M_{\odot}\right)$, related baryonic density $\left(\rho_{\mathrm{DU}}\right)$ and proton fraction $\left(Y_{\mathrm{DU}}\right)$.

\begin{tabular}{l|l|c|c|c|c|c|c|c}
\hline \hline Model & Ref. & $M_{\max } / M_{\odot}$ & $R(\mathrm{~km})$ & $R_{1.44 M_{\odot}}(\mathrm{km})$ & $\varepsilon_{c}\left(\mathrm{fm}^{-4}\right)$ & $M_{\mathrm{DU}} / M_{\odot}$ & $\rho_{\mathrm{DU}}\left(\mathrm{fm}^{-3}\right)$ & $Y_{\mathrm{DU}}$ \\
\hline \multicolumn{1}{|c}{$\vdots$} \\
\hline $\mathrm{DDH} \delta$ & {$[4]$} & 2.142 & 11.138 & 12.587 & 6.412 & 1.876 & 0.569 & 0.138 \\
\hline $\mathrm{DD}-\mathrm{ME} \delta$ & {$[5]$} & 1.958 & 10.241 & 11.836 & 7.714 & 1.793 & 0.765 & 0.140 \\
\hline \hline
\end{tabular}

Notice that the corrected values for the maximum stellar masses modified from 12 to 13 the number of parametrizations that produce neutron stars with maximum mass in the range of $1.93 \leqslant M / M_{\odot} \leqslant 2.05$. They are: BKA20, BKA22, BKA24, BSR8, BSR9, BSR10, BSR11, BSR12, FSUGZ03, G2*, IU-FSU, DD-F, and DD-ME $\delta$. Now, only two parametrizations, TW99 and DDH $\delta$, yield maximum masses above the bands shown in Fig. 1.

Another correction was made in the results of Sec. IIC. Now, the inclusion of hyperons in the DDH $\delta$ parametrization generates, for both sets (SET1 and SET2), maximum masses lower than the range of $1.93 \leqslant M / M_{\odot} \leqslant 2.05$, see Table II. Notice also the same behavior presented by DDH $\delta$ and TW99 parametrizations, namely, the larger reduction in the maximum neutron star mass verified when SET2 is considered.

TABLE II. Main properties of neutron star with hyperonic matter

\begin{tabular}{l|c|c|r|c|c}
\hline \hline couplings & Model & $M_{\max } / M_{\odot}$ & $R(\mathrm{~km})$ & $R_{1.44 M_{\odot}}(\mathrm{km})$ & $\varepsilon_{c}\left(\mathrm{fm}^{-4}\right)$ \\
\hline SET 1 & TW99 & 1.895 & 9.631 & 11.015 & 8.671 \\
SET 2 & TW99 & 1.700 & 10.166 & 11.432 & 7.774 \\
SET 1 & DDH $\delta$ & 1.859 & 10.039 & 11.790 & 7.495 \\
SET 2 & DDH $\delta$ & 1.689 & 10.580 & 12.456 & 7.256 \\
\hline \hline
\end{tabular}




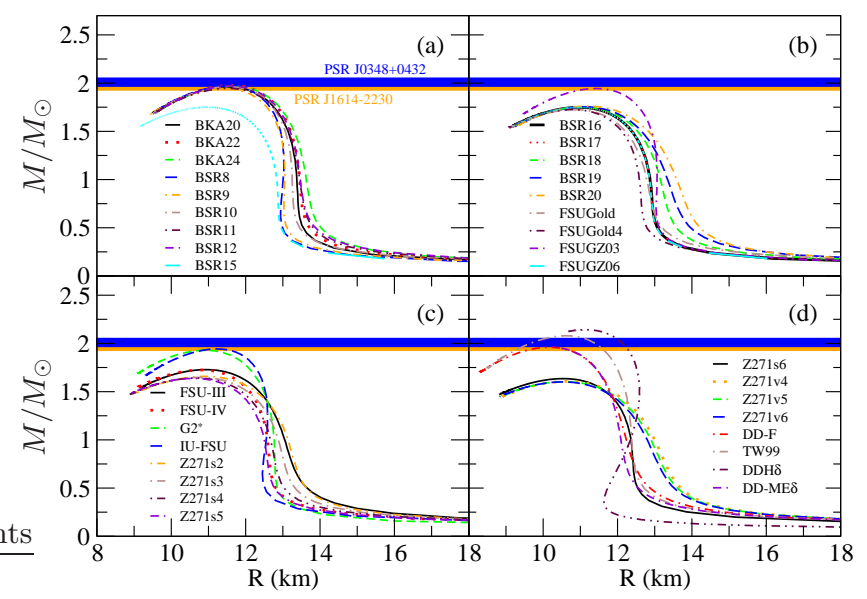

FIG. 1. Neutron star mass-radius relation. Horizontal bands indicate the masses of PSR J1614-2230 [2] (orange) and PSR J038+0432 [3] (blue).

For the sake of completeness, we have also reanalyzed the sound velocity. The corrected curves for the DDH $\delta$ and DD-ME $\delta$ parametrizations are depicted in Fig. 2 as follows. The conclusions drawn in Ref. [1] remain basically the same in this case.

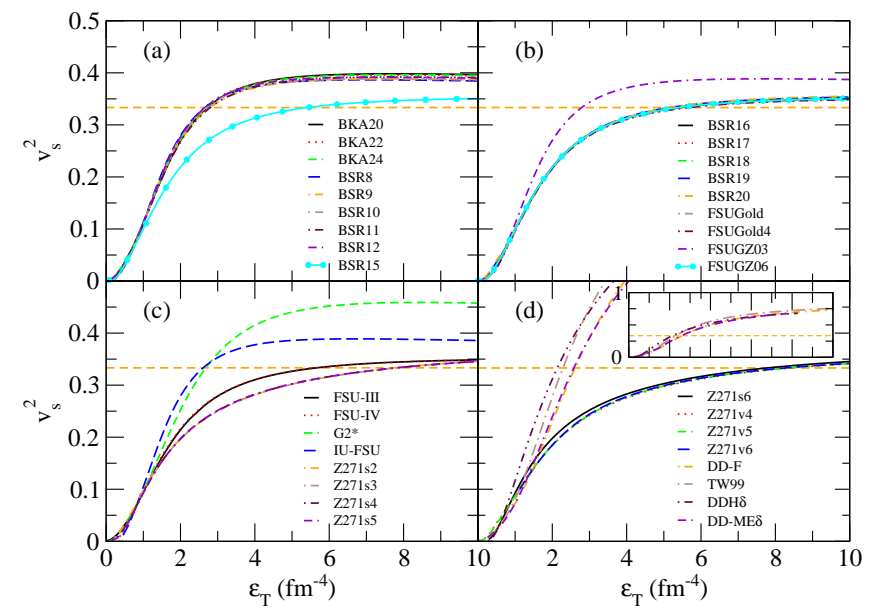

FIG. 2. Squared sound velocity for nuclear matter in beta equilibrium. Dashed line represents the limit $v_{s}^{2}=\frac{1}{3}$.

\section{ACKNOWLEDGMENTS}

This work was partially supported by CNPq under grant 300602/2009-0. O.L. acknowledges support from CNPq. M.D. acknowledges support from Fundação de Amparo à Pesquisa do Estado do Rio de Janeiro (FAPERJ), Grant No. 111.659/2014. We would like to thank Prof. Constança Providência from University of Coimbra, Portugal, for drawing our attention to the mistake in our calculations.

[1] Mariana Dutra, Odilon Lourenço, and Débora P. Menezes, Phys. Rev. C 93, 025806 (2016).

[2] P. B. Demorest, T. Pennucci, S. M. Ransom, M. S. E. Roberts, and J. W. T. Hessels, Nature 467, 1081 (2010).

[3] J. Antoniadis, P. C. C. Freire, N. Wex et al., Science 340, 448 (2013).

[4] T. Gaitanos, M. Di Toro, S. Typel, V. Baran, C. Fuchs, V. Greco, and H. H. Wolter, Nucl. Phys. A 732, 24 (2004).

[5] X. Roca-Maza, X. Viñas, M. Centelles, P. Ring, and P. Schuck Phys. Rev. C 84, 054309 (2011). 


\title{
Stellar properties and nuclear matter constraints
}

\author{
Mariana Dutra ${ }^{1}$, Odilon Lourenço ${ }^{2}$ and Débora P. Menezes $^{3}$ \\ ${ }^{1}$ Departamento de Ciências da Natureza - IHS, Universidade Federal Fluminense, 28895-532 Rio das Ostras, RJ, Brazil \\ ${ }^{2}$ Departamento de Ciências da Natureza, Matemática e Educação, CCA, \\ Universidade Federal de São Carlos, 13600-970 Araras, SP, Brazil \\ ${ }^{3}$ Depto de Física - CFM - Universidade Federal de Santa Catarina, \\ Florianópolis - SC - CP. 476 - CEP 88.040 - 900 - Brazil
}

(Dated: September 16, 2021)

\begin{abstract}
We have analyzed stellar properties of the relativistic mean-field (RMF) parametrizations shown to be consistent with the recently studied constraints related to nuclear matter, pure neutron matter, symmetry energy and its derivatives [Dutra et al., Phys. Rev. C 90, 055203 (2014)]. Our results show that only two RMF parametrizations do not allow the emergence of the direct Urca process, important aspect regarding the evolution of a neutron star. Moreover, among all approved RMF models, fourteen of them produce neutron stars with maximum masses inside the range $1.93 \leqslant M / M_{\odot} \leqslant 2.05$, with $M_{\odot}$ being the solar mass. Only three models yield maximum masses above this range and a discussion on the inclusion of hyperons is presented. Finally, we have verified that the models satisfying the neutron star maximum mass constraint do not observe the squared sound velocity bound, namely, $v_{s}^{2}<1 / 3$, corroborating recent findings. However, the recently proposed $\sigma$-cut scheme can make the RMF models consistent with both constraints depending on the isoscalar-vector interaction of each parametrization.
\end{abstract}

PACS numbers: 21.30.Fe, 21.65.Cd, 26.60.Kp, 24.10.Jv

\section{INTRODUCTION}

Historically, around 1930, the first nuclear physics model known as the liquid drop model [1] and the semi-empirical mass formula presented by Bethe and Weizsäcker [2], established the grounds for the study of nuclei properties and nuclear structure. These two models are so close to each other in basic ideas, that their nomenclature is very often mixed up. Both are parameter dependent models and their underlying fitting procedure was later used in the development of many other models. Nowadays, around 500 hundred nonrelativistic (Skyrmetype) and relativistic models are available in the literature and largely used in describing different features, from nuclear to hadron spectroscopy, from heavy-ion collisions to neutron star properties. The vast majority of the models are parameter dependent based on the fitting of nuclear bulk matter. These phenomenological models generally rely on the calculation of equations of state (EoS), which relate pressure, energy density and temperature at a given particle number density subject to nuclear forces.

In the last years, detailed analyses of nonrelativistic and relativistic models were performed $[3,4]$. From such studies, it was suggested that the proliferation of models and the production of new parameter sets with a limited range of application should not be encouraged. To be more specific, in [3], 240 different Skyrme model parameterizations were confronted with experimentally and empirically derived constraints and only 16 were shown to satisfy all of them. Other 263 relativistic mean-field (RMF) parametrizations were also analysed in [4] and once again, only a small number of them, 35 to be exact, were shown to satisfy adequately chosen constraints. It is important to say that three different sets of constraints were used in [4], all of them related to symmetric nuclear matter, pure neutron matter, symmetry energy and its derivatives. They differed one from the other in the choice of validity ranges of certain quantities and in the level of restriction. In a recent study [5], the authors also constrained the EoS of Skyrme and RMF models to those predicted from chiral effective field theory including twoand three-nucleon forces effects.

In [4], the relativistic models were divided into 7 families, namely, linear finite range models (Walecka-type models [6], type 1), non-linear $\sigma$ models (Boguta-Bodmer models [7], type 2), non-linear $\sigma$ and $\omega$ models with a self-quartic interaction in the $\omega$ field (type 3 ), non-linear $\sigma$ and $\omega$ terms and cross terms involving these fields (type 4), density dependent models [8] with couplings adjusted to nuclear properties (type 5), non-linear point coupling models [9] (type 6) and models with $\delta$ mesons (type 7 ). Thirty of the approved models are of type 4 , two are of type 5 , one of type 6 , and two of type 7 being both density dependent. In the present work, we go one step further and confront all the approved models with observational astrophysical properties. Until not very long ago (2010), all mean-field models adapted to stellar conditions (charge neutrality and chemical equilibrium) that resulted in maximum stellar masses larger than $1.44 M_{\odot}$ were acceptable. After two massive stars were discovered, namely, the PSR J1614-2230 with mass of $(1.97 \pm 0.04) M_{\odot}$ [10], and the PSR J0348+0432 with mass of $(2.01 \pm 0.04) M_{\odot}$ [11], many parameter dependent models were re-tuned so that they could describe maximum masses in these ranges. Other mechanisms capable of stiffening the EoS and hence increasing the maximum stellar mass were also proposed as in [12-14], but we will discuss them after we present our results. 
The aim of the present work is to check whether the 35 RMF parametrizations that were shown to satisfy the nuclear matter constraints in [4] also satisfy the criterion of producing massive stars. The same EoS are also used to investigate the direct Urca process and to calculate the sound velocity.

\section{FORMALISM AND RESULTS}

To obtain neutron star macroscopic properties, the necessary steps are the following ones: 1) the EoS for hadronic matter is joined with the EoS for free leptons; 2) the conditions of charge neutrality and chemical equilibrium are enforced; 3) the BPS equation of state [15] for low densities is added to the EoS for hadrons and leptons; 4) the resulting EoS is used as input to the TolmanOppenheimer-Volkoff equations [16], which are the differential equations for the structure of a static, spherically symmetric star in hydrostatic equilibrium. All these steps are well known and the details are given in many papers and books, as for instance, in $[17,18]$. Next, only electrons and muons are considered in the leptonic EoS since we just consider the zero temperature deleptonized phase of the stellar evolution.

As a remark, we remind the reader that clusterized and possibly the pasta phase matters can also be present in the inner crust of the star. However, in the present calculation, it is important to point out that after we join the BPS EoS, used to describe the outer crust, to the hadronic one obtained from the RMF models, the TOV equations are solved. In doing so, an interpolation is used and the exact values in between these two EoSs loose their strict meaning. In our calculations, the lowest baryonic density obtained from the relativistic models is approximately $\rho=0.05 \mathrm{fm}^{-3}$ and the BPS points start respectively at $\rho=0.008907 \mathrm{fm}^{-3}$ (baryonic density), $\varepsilon=0.04253 \mathrm{fm}^{-4}$ (energy density) and $p=0.00006987 \mathrm{fm}^{-4}$ (pressure) and go up to a very low baryonic density, namely $\rho=0.1581 \times 10^{-10} \mathrm{fm}^{-3}$, and corresponding energy density and pressure. Any different matter structure existing in between these two densities is washed out when the TOV equations are used. Hence, the detailed structure of the inner crust is not relevant for the present analysis. In our analysis, we start with nucleons only in the hadronic sector and the results are presented next.

\section{A. Nucleonic matter}

In Table I we display some of the neutron star properties obtained from the solution of the TOV equations after the 35 models approved in [4] are used as input. The first 30 results correspond to models of type 4, described in the Introduction and the respective Lagrangian den- sities can be obtained from the references listed in the second column. The last 4 results come from density dependent models and in the last 2, the scalar isovector $\delta$ meson is also included. Regarding the only pointcoupling model approved according to Ref. [4], the FA3 one, we remark that due to its very particular behavior at high density regime, namely, a fall in the curve $p \times \varepsilon$ at around $\varepsilon=4.1 \mathrm{fm}^{-4}$ ( $p$ is the pressure and $\varepsilon$ is the energy density), it was not possible to generate a mass radius curve indicating a maximum mass and, consequently, no other quantities presented in Table I. Therefore, we have discarded such a model from our analysis. In Fig. 1, the corresponding mass radius curves are plotted, alongside with the bands that represent astrophysical constraints of the order of $2 M_{\odot}$ on the stellar masses. From these results, one can immediately see that the models BKA20, BKA22, BKA24, BSR8, BSR9, BSR10, BSR11, BSR12, FSUGZ03, G2*, IU-FSU, and DD-F are inside the maximum mass constraint of $[10,11]$, and that only 3 models yield maximum masses above the bands shown in Fig. 1, namely, TW99, $\mathrm{DDH} \delta$ and $\mathrm{DD}-\mathrm{ME} \delta$. It is a very well known fact that hyperons are expected to exist in the core of neutron stars and that when they are included in the EoS, it becomes softer and the corresponding maximum stellar mass decreases considerably. Hence, just these 3 models will be selected for the study of hyperonic matter. Before we proceed to perform this analysis, we look at three other important aspects related to neutron stars: their radii, the direct Urca process (DU) and the sound velocity.

As far as neutron star radii are considered, there is a bit of controversy in their acceptable values, as discussed in the literature. In Ref. [31], for example, the authors constrained the radii of the canonical $1.44 M_{\odot}$ neutron star to the range of $9.7 \leqslant R_{1.44 M_{\odot}} \leqslant 13.9 \mathrm{~km}$, based on a chiral effective theory analysis. In our study, we observe that all values are within such a range (see the fifth column of Table I). In Ref. [32], on the other hand, a limit of $12 \mathrm{~km}$ is found for $R_{1.44 M_{\odot}}$, while in Ref. [33] the limit is $13.1 \mathrm{~km}$. Another calculation, discussed in Ref. [34], based on a Bayesian analysis, results in radii of all neutron stars in the range of $10.9 \mathrm{~km}$ and $12.7 \mathrm{~km}$, while even significantly smaller radii are predicted in Refs. [35, 36], where the ranges found are of $9.1_{-1.5}^{1.3} \mathrm{~km}$ and $9.4 \pm 1.2 \mathrm{~km}$, respectively, obtained from analyses of five quiescent low-mass x-ray binaries. Finally, it is also possible to infer neutron star radii from the knowledge of the symmetry energy slope at the saturation density $\left(\rho_{o}\right)$ due to the correlation between such quantities, as suggested in Refs. [37, 38]. Some of these ranges and other ones extracted from other recent publications are summarized in Fig. 10 of Ref. [39]. As one can see, definite values for the neutron star radii are not yet established, and the results obtained from the RMF parametrizations analyzed in the present work (most of them in the range of $12 \leqslant R_{1.44 M_{\odot}} \leqslant 13 \mathrm{~km}$ ) are compatible with several of the aforementioned predictions. 
TABLE I. Neutron star main properties: maximum stellar masses in terms of $M_{\odot}\left(M_{\max } / M_{\odot}\right)$, the radius of the corresponding star $(R)$, the radius of a star with a $1.44 M_{\odot}$ mass $\left(R_{1.44 M_{\odot}}\right)$ and the central energy density of the maximum mass star $\left(\varepsilon_{c}\right)$. Properties related to the direct Urca process: mass of the star in terms of $M_{\odot}$ at the onset of the DU process $\left(M_{\mathrm{DU}} / M_{\odot}\right)$, related baryonic density $\left(\rho_{\mathrm{DU}}\right)$ and proton fraction $\left(Y_{\mathrm{DU}}\right)$.

\begin{tabular}{|c|c|c|c|c|c|c|c|c|}
\hline Model & Ref. & $M_{\max } / M_{\odot}$ & $R(\mathrm{~km})$ & $R_{1.44 M_{\odot}}(\mathrm{km})$ & $\varepsilon_{c}\left(\mathrm{fm}^{-4}\right)$ & $M_{\mathrm{DU}} / M_{\odot}$ & $\rho_{\mathrm{DU}}\left(\mathrm{fm}^{-3}\right)$ & $Y_{\mathrm{DU}}$ \\
\hline BKA20 & {$[19]$} & 1.960 & 11.522 & 13.191 & 6.177 & 1.065 & 0.311 & 0.133 \\
\hline BKA22 & {$[19]$} & 1.975 & 11.601 & 13.262 & 6.095 & 1.000 & 0.294 & 0.133 \\
\hline BKA24 & {$[19]$} & 1.968 & 11.608 & 13.367 & 6.160 & 0.911 & 0.271 & 0.132 \\
\hline BSR8 & {$[20]$} & 1.969 & 11.503 & 12.970 & 6.090 & 1.410 & 0.405 & 0.135 \\
\hline BSR9 & {$[20]$} & 1.944 & 11.419 & 12.958 & 6.240 & 1.313 & 0.385 & 0.135 \\
\hline BSR10 & {$[20]$} & 1.963 & 11.533 & 13.108 & 6.137 & 1.149 & 0.335 & 0.134 \\
\hline BSR11 & {$[20]$} & 1.946 & 11.504 & 13.208 & 6.264 & 0.980 & 0.294 & 0.133 \\
\hline BSR12 & {$[20]$} & 1.970 & 11.580 & 13.252 & 6.160 & 1.011 & 0.300 & 0.133 \\
\hline BSR15 & {$[20]$} & 1.750 & 10.969 & 12.483 & 6.844 & 1.250 & 0.420 & 0.136 \\
\hline BSR16 & {$[20]$} & 1.748 & 10.968 & 12.494 & 6.893 & 1.231 & 0.414 & 0.136 \\
\hline BSR17 & {$[20]$} & 1.750 & 10.989 & 12.494 & 6.902 & 1.119 & 0.372 & 0.135 \\
\hline BSR18 & {$[20]$} & 1.751 & 11.040 & 12.662 & 6.873 & 1.028 & 0.338 & 0.134 \\
\hline BSR19 & {$[20]$} & 1.754 & 11.102 & 12.773 & 6.828 & 0.923 & 0.303 & 0.133 \\
\hline BSR20 & {$[20]$} & 1.760 & 11.194 & 12.972 & 6.772 & 0.841 & 0.267 & 0.132 \\
\hline FSU-III & {$[21]$} & 1.728 & 10.934 & 12.502 & 7.011 & 1.058 & 0.362 & 0.135 \\
\hline FSU-IV & {$[21]$} & 1.725 & 10.797 & 12.220 & 7.061 & 1.477 & 0.572 & 0.138 \\
\hline FSUGold & {$[22]$} & 1.725 & 10.842 & 12.337 & 7.090 & 1.304 & 0.467 & 0.136 \\
\hline FSUGold4 & {$[23]$} & 1.725 & 10.788 & 12.224 & 7.110 & 1.479 & 0.572 & 0.138 \\
\hline FSUGZ03 & {$[24]$} & 1.944 & 11.418 & 12.963 & 6.240 & 1.314 & 0.385 & 0.135 \\
\hline FSUGZ06 & {$[24]$} & 1.748 & 10.961 & 12.494 & 6.893 & 1.244 & 0.419 & 0.136 \\
\hline $\mathrm{G}^{*}{ }^{*}$ & {$[25]$} & 1.929 & 10.907 & 12.551 & 6.959 & 1.197 & 0.390 & 0.135 \\
\hline IU-FSU & {$[26]$} & 1.943 & 11.228 & 12.563 & 6.348 & 1.776 & 0.614 & 0.138 \\
\hline Z271s2 & {$[27]$} & 1.658 & 10.910 & 12.464 & 7.098 & 1.079 & 0.366 & 0.135 \\
\hline Z271s3 & [27] & 1.647 & 10.751 & 12.238 & 7.301 & 1.301 & 0.488 & 0.137 \\
\hline Z271s4 & {$[27]$} & 1.640 & 10.669 & 12.070 & 7.347 & 1.470 & 0.637 & 0.138 \\
\hline Z271s5 & {$[27]$} & 1.637 & 10.590 & 11.952 & 7.424 & 1.562 & 0.789 & 0.140 \\
\hline Z271s6 & {$[27]$} & 1.635 & 10.534 & 11.859 & 7.469 & 1.607 & 0.934 & 0.140 \\
\hline Z271v4 & {$[27]$} & 1.606 & 10.639 & 12.085 & 7.590 & 1.272 & 0.500 & 0.137 \\
\hline Z271v5 & {$[27]$} & 1.603 & 10.572 & 11.984 & 7.667 & 1.501 & 0.748 & 0.140 \\
\hline Z271v6 & {$[27]$} & 1.601 & 10.514 & 11.902 & 7.743 & 1.585 & 1.028 & 0.141 \\
\hline DD-F & {$[28]$} & 1.960 & 10.173 & 11.880 & 7.981 & - & - & - \\
\hline TW99 & {$[8]$} & 2.080 & 10.613 & 12.254 & 7.239 & - & - & - \\
\hline $\mathrm{DDH} \delta$ & [29] & 2.540 & 12.460 & 13.282 & 4.917 & 2.000 & 0.569 & 0.138 \\
\hline DD-ME $\delta$ & {$[30]$} & 2.433 & 11.657 & 12.296 & 5.520 & 2.112 & 0.766 & 0.140 \\
\hline
\end{tabular}

The direct Urca (DU) process, $n \rightarrow p+e^{-}+\bar{\nu}_{e}[40]$ sheds light on an important aspect of the evolution of neutron stars. The cooling of the star by neutrino emission can occur relatively fast when the proton fraction exceeds a critical value $x_{\mathrm{DU}}[40]$, evaluated in terms of the leptonic fraction as $[28] x_{\mathrm{DU}}=\frac{1}{1+\left(1+x_{e}^{1 / 3}\right)^{3}}$, where $x_{e}=\rho_{e} /\left(\rho_{e}+\rho_{\mu}\right)$ is the electron leptonic fraction, with $\rho_{e}$ and $\rho_{\mu}$ being the densities of electrons and muons, respectively. Cooling rates of neutron stars [41] seem to indicate that this fast cooling process does not occur, except in stars with a mass larger than $1.5 M_{\odot}[28]$. Hence, from the results in Table I, one can see that all selected models could give rise to the DU process. In the same table, we show the results for the star mass obtained when the proton fraction crosses the line of the electron fraction. We also display the results of the corresponding baryonic density and the proton fraction. While most of the analyzed models cross the line that establishes the onset of the DU process when the proton fraction is of the order of 0.14 , the densities can vary substantially. Most models cross this line for very low densities, but there are some exceptions, when the crossing takes place at around half the saturation density or even at higher densities (Z271s5, Z271s6, Z271v5, Z271v6, DD-ME $\delta$ ). In [37], it is shown that the DU process is related to the density dependence of the symmetry energy and hence, to the isovector channel of the EoS and to its slope: the larger the slope of the symmetry energy, which corresponds to a harder symmetry energy, the smaller the onset density because larger proton fractions are favored in the system. Hence, our analyses of 32 of the models show that the fast cooling can indeed take place if the stars are described by them. On the other hand, the 


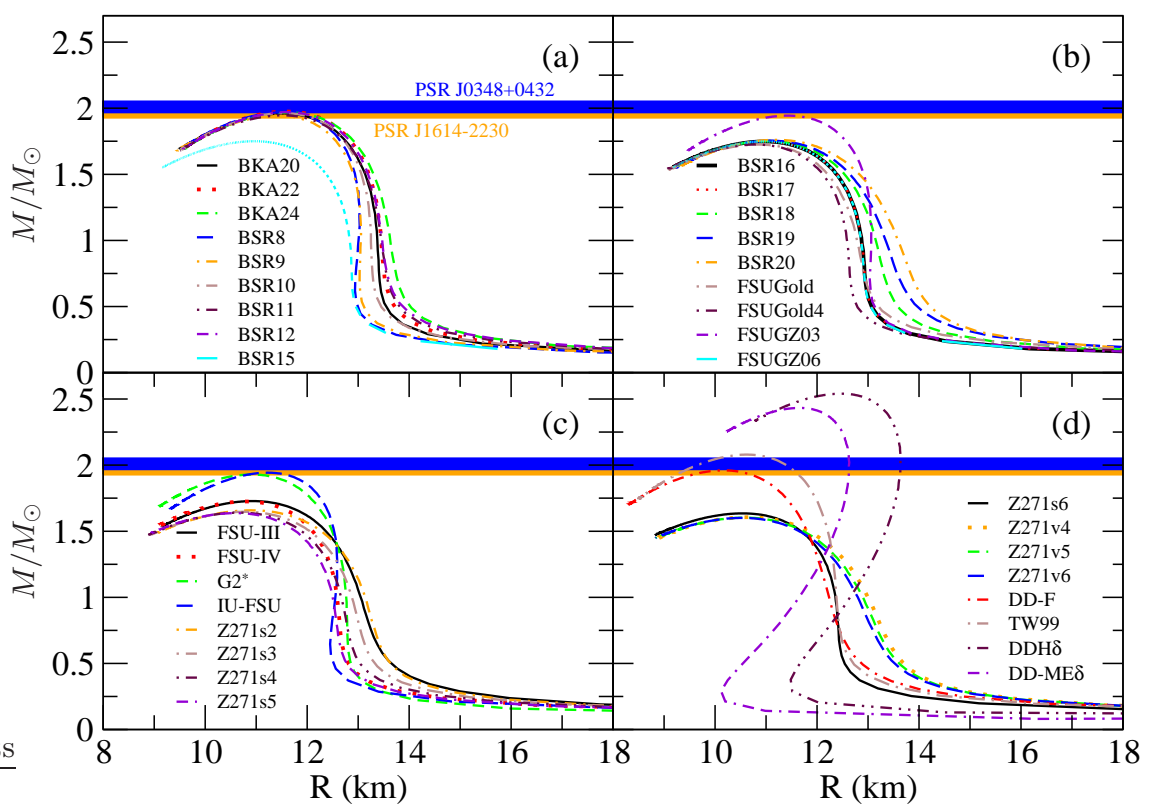

FIG. 1. (Color online) Neutron star mass-radius relation. Horizontal bands indicate the masses of PSR J1614-2230 [10] (orange) and PSR J038+0432 [11] (blue).

parametrizations DD-F and TW99 do not support this possibility because the proton fraction never crosses the electron leptonic fraction.

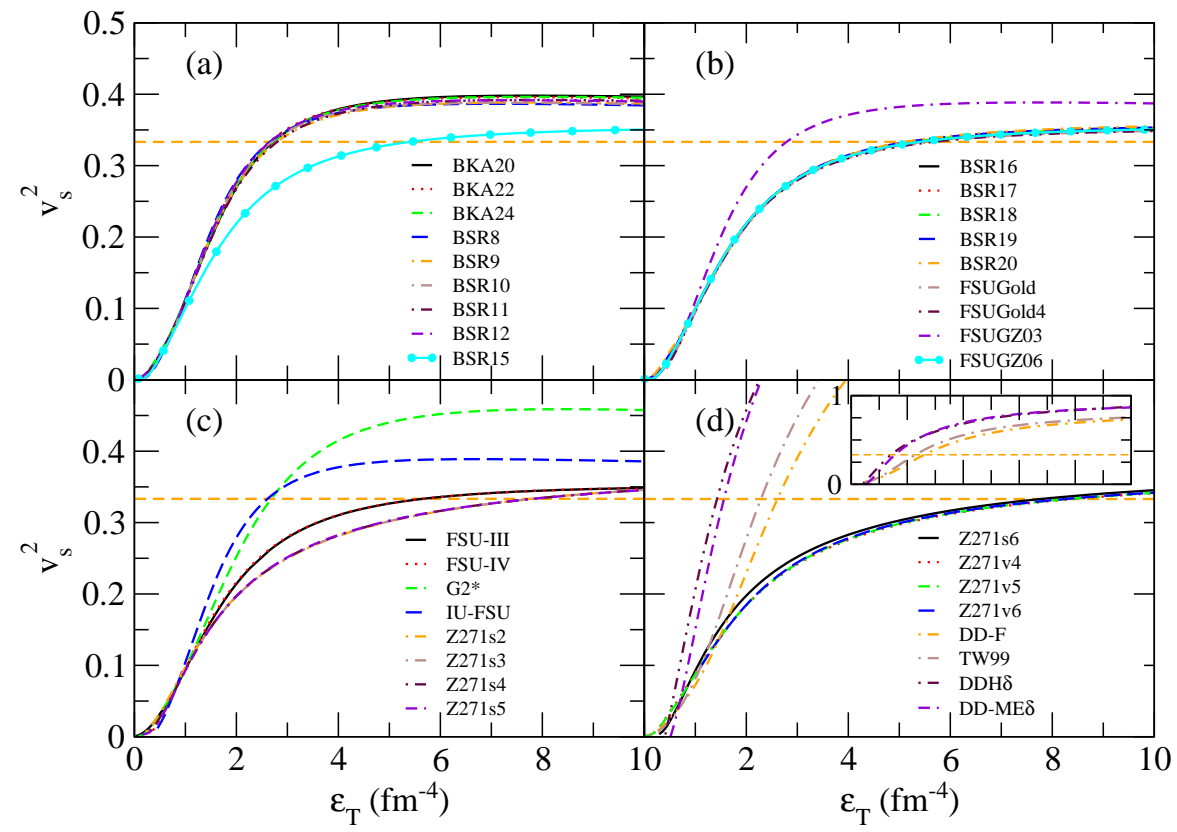

FIG. 2. (Color online) Squared sound velocity for nuclear matter in beta equilibrium. Dashed line represents the limit $v_{s}^{2}=\frac{1}{3}$.

\section{B. Sound velocity constraint}

We now move our attention to the calculation of the sound velocity, which can be easily obtained from the EoS, since for matter in beta equilibrium it is given by $v_{s}^{2}=d p_{T} / d \varepsilon_{T}$, where $p_{T}$ and $\varepsilon_{T}$ are the total pressure and total energy density of the system, respectively. In Fig. 2 we show the behaviour of the squared sound velocity as a function of the total energy density. Causality constrains $v_{s}$ to the light velocity in vacuum, $c$, which we take as 1 in the present work. Although only four models (DD-F, TW99, DDH $\delta$ and DD-ME $\delta$ ) reach quite high 
values, they never exceed 0.9 , as one can see in the inset of Fig. 2(d), and even though, only at very high energy densities, around $\varepsilon_{T}=10 \mathrm{fm}^{-4}$, which are higher than the central energy densities in stars described by these models, as seen in Table I.

We still remark here that results derived from QCD [42] and also according to Ref. [43], another limitation to the sound velocity, given by $v_{s}^{2}=1 / 3$, is confirmed by several classes of strongly coupled theories with gravity duals. However, if one examines, for instance, Fig. 5 of Ref. [13], where a study on the effects of meson-hyperon coupling constants on the onset of hyperons in dense nuclear matter is performed, one can see that at the onset of every individual hyperon, the sound velocity shows a peak, decreases and starts increasing again. If instead of the appearance of new hyperons, the EoS suffered a transition to another phase, which could be a mixed phase of hyperons and quarks or a phase containing only quarks (see Ref. [44], as an example), the decrease in the sound velocity could be even more abrupt. The conclusions drawn in Ref. [43] refer to matter in one phase only. Had the authors considered a phase transition (not discarded by observational constraints), the squared sound velocity could reach values higher than $1 / 3$, decrease considerably at the appearance of the new phase, and then increase up to values around $1 / 3$, related to the limit imposed by QCD.

Our results show that most of the models reach values larger than $1 / 3$ at energy densities below the stellar central energy density. The exceptions are the class of models Z271, where the squared sound velocity reaches $1 / 3$ at energy densities slightly higher than the corresponding star central energy density. We do not have enough statistics to produce a histogram as the one shown in [43], but our findings corroborates the statement in [43], namely, that are unlikely models with acceptable behavior at low densities capable of producing maximum masses around $2 M_{\odot}$, and, simultaneously, satisfying the bound $v_{s}^{2}<1 / 3$, since here we are not taking into account the possibility of the system undergoing phase transitions. Notice that in the case of the models Z271, compatible with sound velocity bound, the maximum masses are all lower than $2 M_{\odot}$, as we can see in Table I.

Based on these results, let us examine those parametrizations in which the maximum neutron star mass is below the minimum value of $1.93 M_{\odot}$ established in Ref. [10], namely, BSR15-BSR20, FSU-III, FSU-IV, FSUGold, FSUGold4, FSUGZ06, Z271s2-Z271s6, and Z271v4-Z271v6. A valid attempt to make such parametrizations consistent with the the constraint of $1.93 \leqslant M / M_{\odot} \leqslant 2.05$ for the maximum neutron star mass, is to modify the models at high density limit $\left(\rho>\rho_{o}\right)$ in such way that their saturation properties are not altered, i. e., quantities such as binding energy, saturation density, effective mass, incompressibility, symmetry energy, etc., are kept the same for each parametrization. This procedure ensures that results presented by such models under nuclear matter constraints at $\rho=\rho_{0}$, investigated in Ref. [4], remain valid.

In Ref. [14], the authors proposed the called $\sigma$-cut scheme in which they add in the $U(\sigma)$ potential of the RMF models, the function $\Delta U(\sigma)=\alpha \ln \{1+\exp [\beta(f-$ $\left.\left.\left.f_{\text {s.core }}\right)\right]\right\}$, where $f=g_{\sigma} \sigma / M_{N}$ and $f_{\text {s.core }}=f_{0}+c_{\sigma}\left(1-f_{0}\right)$. The value of $f$ at the saturation density is $f_{0} ; \alpha, \beta$ and $c_{\sigma}$ are constants; $g_{\sigma}$ regulates the strength of the attractive interaction, denoted by the scalar field $\sigma$; and $M_{N}$ is the nucleon rest mass. In this scheme, it is possible to avoid the decreasing in the density dependence of the effective nucleon mass, $M^{*}=M_{N}-g_{\sigma} \sigma$, through the function $\Delta U(\sigma)$. Such a decreasing is present in the original RMF models where $\Delta U(\sigma)=0$. It is responsible by softening the EoS (since the attraction is increasing), avoiding the system to reach higher values for the maximum neutron star mass. According to Ref. [14], the necessary condition that needs to be satisfied in order to prevent $M^{*}$ of decreasing in the $\sigma$-cut scheme, is expressed in terms of the constants $\alpha$ and $\beta$ by $\alpha \beta^{2} \gg 6 \times 10^{-3} M_{N}^{4} \rho / \rho_{o}$. Since this condition is verified, the constant $c_{\sigma}$ controls at which density, denoted by $\rho_{*}$, the decreasing in $M^{*}$ stops. In order to ensure that the bulk parameters of the models are preserved, it is natural to choose $c_{\sigma}$ in such way that $\rho_{*}>\rho_{0}$.

Let us consider here the values of $\alpha=4.822 \times 10^{-4} M_{N}^{4}$ and $\beta=120$ used in Ref. [14], and apply the $\sigma$-cut scheme in the models presenting $M<1.93 M_{\odot}$. By properly choosing the $c_{\sigma}$ values, it is possible to generate the neutron star mass-radius relation depicted in Fig. 3.

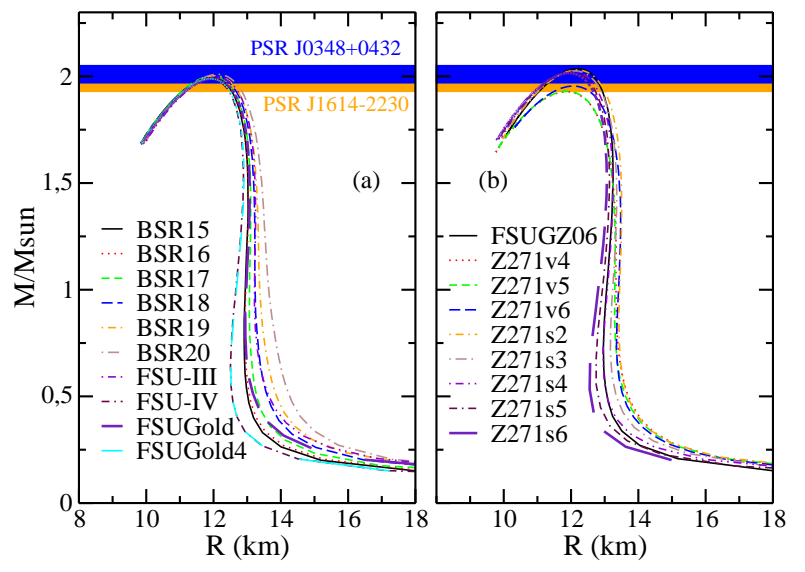

FIG. 3. (Color online) $M \times R$ for models presenting $M<1.93 M_{\odot}$ in Table I. Here we used (see text) $c_{\sigma}=0.35$ and 0.31 , respectively, for the BSR and FSU parametrizations. For Z271v4, Z271v5 and Z271v6 ones, we chose $c_{\sigma}=0.145$, 0.14 and 0.12 , respectively.

As one can see, the maximum neutron star mass constraint is now satisfied. Moreover, if we further investigate these modified parametrizations under the sound velocity bound proposed in Ref. [43], we see that within the $\sigma$-cut scheme, they are now consistent with such a constraint, as showed in Fig. 4(a).

However, we see in Fig. 4(b) that for the Z271s 


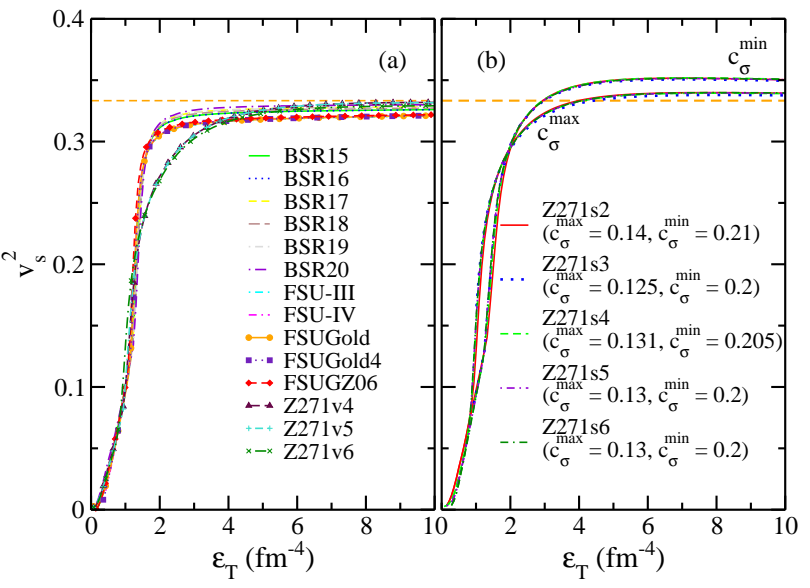

FIG. 4. (Color online) Squared sound velocity for nuclear matter in beta equilibrium for (a) BSR, FSU, Z271v, and (b) Z271s parametrizations in the $\sigma$-cut scheme. Dashed line represents the limit $v_{s}^{2}=\frac{1}{3}$. The values of $c_{\sigma}$ for models in panel (a) are the same used in Fig. 3. In panel (b), the values of $c_{\sigma}^{\min }$ and $c_{\sigma}^{\max }$ are those that produce maximum neutron star mass of $1.93 M_{\odot}$ and $2.05 M_{\odot}$, respectively.

parametrizations the sound velocity bound is not verified even within the $\sigma$-cut scheme. This is true for any $c_{\sigma}$ inside a range of $c_{\sigma}^{\min } \leqslant c_{\sigma} \leqslant c_{\sigma}^{\max }$, where $c_{\sigma}^{\text {min }}\left(c_{\sigma}^{\max }\right)$ is the value that produces a maximum neutron star mass equal to $1.93 M_{\odot}\left(2.05 M_{\odot}\right)$ for the Z271s models. In order to try to understand the origin of such a result, we point out to the reader that models analyzed in Fig. 4 are described by the Lagrangian density given by $\mathcal{L}=\mathcal{L}_{\text {Walecka }}-\frac{A}{3} \sigma^{3}-\frac{B}{4} \sigma^{4}+$ $\frac{C}{4}\left(g_{\omega}^{2} \omega_{\mu} \omega^{\mu}\right)^{2}+\mathcal{L}_{\sigma \omega \rho}$, where $\mathcal{L}=\mathcal{L}_{\text {Walecka }}$ is the very known Lagrangian density of the linear Walecka model (see Ref. [4]), and $\mathcal{L}_{\sigma \omega \rho}=g_{\sigma} g_{\omega}^{2} \sigma \omega_{\mu} \omega^{\mu}\left(\alpha_{1}+\frac{1}{2} \alpha_{1}{ }^{\prime} g_{\sigma} \sigma\right)+$ $g_{\sigma} g_{\rho}^{2} \sigma \vec{\rho}_{\mu} \vec{\rho}^{\mu}\left(\alpha_{2}+\frac{1}{2} \alpha_{2}{ }^{\prime} g_{\sigma} \sigma\right)+\frac{1}{2} \alpha_{3}{ }^{\prime} g_{\omega}^{2} g_{\rho}^{2} \omega_{\mu} \omega^{\mu} \vec{\rho}_{\mu} \vec{\rho}^{\mu}$. For the Z271s models not satisfying the sound velocity bound, there is no other isoscalar-vector interaction term besides the one regulated by the coupling constant $C$. In these models, $\alpha_{1}=\alpha_{1}{ }^{\prime}=\alpha_{3}{ }^{\prime}=0\left(\right.$ also $\left.\alpha_{2}=0\right)$. All the other models consistent with the limit of $v_{s}^{2}$ in Fig. 4(a), present at least the term whose strength is controlled by the constant $\alpha_{3}{ }^{\prime}$, besides that one presenting $C$. Actually, this is the case for the Z271v models. They have $\alpha_{1}=\alpha_{1}{ }^{\prime}=\alpha_{2}=\alpha_{2}{ }^{\prime}=0$, but only $C$ and $\alpha_{3}{ }^{\prime}$ not equal to zero. One can see that the isoscalar-vector interaction seems to play an important role in the phenomenology of RMF models, concerning the simultaneous consistency of the sound velocity bound of $v_{s}^{2}=\frac{1}{3}$, verified in Ref. [43], and the maximum neutron star mass constraint given by $1.93 \leqslant M / M_{\odot} \leqslant 2.05$.

\section{Hyperonic matter}

We now consider the case when hyperons are also included in the EoS. At this point, we remind the reader that the inclusion of hyperons in hadronic RMF models is very important in the context of compact stars, since simple energetic considerations $[17,45]$ suggest they should be present at the high density regime that such objects naturally exhibit. For any hadronic system, as the baryon density increases, the Fermi level rises sufficiently to start allowing for the emergence of the baryonic components presenting strangeness, i. e., hyperons such as $\Lambda, \Sigma^{+}, \Sigma^{0}, \Sigma^{-}, \Xi^{0}$, and $\Xi^{-}$. Due to the inclusion of these hyperons, the equation of state becomes softer with a consequent reduction of the maximum neutron star mass $[17,45]$. However, the current status of this observational quantity points out to the opposite direction, i. e., recent findings of the massive stars PSR J1614-2230 and PSR J0348+0432 show that old predictions for the maximum neutron star mass around $1.44 M_{\odot}$, are now replaced by the range of $1.93 \leqslant M / M_{\odot} \leqslant 2.05$, as we considered in our work. This fact leads to the problem that the EoS of the models needs to be stiffer in order to predict higher masses and this is not the case if hyperons are included. This contradiction is known in the literature as the hyperon-puzzle, and nowadays, many efforts have been directed to this subject in order to circumvent it as seen in Refs. [46, 47].

Regarding the meson-hyperon couplings, they are responsible for large differences in similar calculations for stellar masses with hyperons $[12,13,17]$. It is not the purpose of the present work to discuss this point in detail since a comprehensive analysis is not simple [47], but we make two choices so that the results can be better understood. We define the ratio between the mesonhyperon and the meson-nucleon couplings as $\chi_{\sigma}=\frac{g_{Y \sigma}}{g_{N \sigma}}$, $\chi_{\omega}=\frac{g_{Y \omega}}{g_{N \omega}}, \chi_{\rho}=\frac{g_{Y \rho}}{g_{N \rho}}, \chi_{\delta}=\chi_{\sigma}$, where $Y$ represents any of the six lowest mass hyperons and $g_{N i}$ represents the coupling of the nucleon with any of the four fields and it is density dependent in the cases we study next. The dependence with the density varies according to model used and they can be found in the original references given in Table I.

The first scenario we examine is the one called universal coupling [48], given by $\chi_{\sigma}=\chi_{\omega}=\chi_{\rho}=\chi_{\delta}=1$ and we next call it SET 1. For the second scenario, considered more realistic because it is based on hypernuclei experimental values [17], we use $\chi_{\sigma}=\chi_{\delta}=0.7$, $\chi_{\omega}=\chi_{\rho}=0.783$ and we call it SET 2. The results are displayed in Table II. One can see that the maximum

TABLE II. Neutron star main properties with hyperonic matter

\begin{tabular}{l|c|c|r|c|c}
\hline \hline couplings & Model & $M_{\max } / M_{\odot}$ & $R(\mathrm{~km})$ & $R_{1.44 M_{\odot}(\mathrm{km})}$ & $\varepsilon_{c}\left(\mathrm{fm}^{-4}\right)$ \\
\hline SET 1 & TW99 & 1.895 & 9.631 & 11.015 & 8.671 \\
\hline SET 2 & TW99 & 1.700 & 10.166 & 11.432 & 7.774 \\
\hline \hline SET 1 & DDH $\delta$ & 2.302 & 11.307 & 12.523 & 5.781 \\
\hline SET 2 & DDH $\delta$ & 2.364 & 12.491 & 13.532 & 4.485 \\
\hline \hline SET 1 & DD-ME $\delta$ & 2.173 & 10.420 & 11.283 & 7.005 \\
\hline SET 2 & DD-ME $\delta$ & 2.254 & 11.473 & 12.352 & 5.681 \\
\hline \hline
\end{tabular}


masses obtained with SET 2 are higher if the $\delta$ meson is present in the model and lower otherwise. The inclusion of hyperons decrease the maximum mass as compared with the results with nucleons only by $9 \%$ to $19 \%$, depending on the choice for the meson-hyperon constants. It is important to stress that many other choices would be possible, but in some cases the baryon effective masses decrease very rapidly and the EoS stops converging before the maximum stellar masses are attained. The convergence of the codes should always be carefully checked. In the cases under examination, the radii of the canonical $1.44 M_{\odot}$ star remain within the expected range.

\section{FINAL REMARKS}

In the present paper we have revisited the RMF models that were shown to satisfy several nuclear matter constraints in [4] and confronted them with astrophysical constraints. From the 35 analyzed models with nucleonic matter included, only the BKA20, BKA22, BKA24, BSR8, BSR9, BSR10, BSR11, BSR12, FSUGZ03, G2*, IU-FSU, and DD-F describe neutron stars with maximum mass in the range of $1.93 \leqslant M / M_{\odot} \leqslant 2.05[10$, 11]. Only three models can sustain maximum masses larger than $2.05 M_{\odot}$ when nucleonic matter is considered (TW99, DDH $\delta, \mathrm{DD}-\mathrm{ME} \delta$ ) and only two still reach this value once hyperons are included (DDH $\delta, \mathrm{DD}-\mathrm{ME} \delta$ ). These two models have density dependent couplings and $\delta$ mesons in their Lagrangian density.

A possible alternative to make the EoS stiffer without altering its properties below nuclear matter saturation density was proposed recently in [14]. Such an alternative could be used in order to save the RMF models presenting a maximum mass below $1.93 M_{\odot}$, inside the constraint of $1.93 \leqslant M / M_{\odot} \leqslant 2.05$. The idea is to force the $\sigma$ self-interaction potential to rise sharply around densities just a bit larger than nuclear saturation density, resulting in the increase of the maximum possible stellar mass. As an example, the authors of [14] use the FSUGold model [22], one of the models approved in our extensive analysis with stellar macroscopic properties shown in Table I. The maximum mass was increased from $1.72 M_{\odot}$ to $2.01 M_{\odot}$. Another more standard possibility is to consider also strange meson fields in the Lagrangian density $[12,13]$ and choose the hyperon-meson couplings such that the appearance of strange hyperons are pushed toward high densities. It is also important to remind the reader that a particular procedure of modifying RMF models in order to make them appropriate to describe neutron stars, without changing their bulk parameters at the saturation density, was proposed in Ref. [49] a long time ago. This procedure is basically supported by the inclusion of quartic self-interactions of the vector-isoscalar and vector-isovector fields related to $\omega$ and $\rho$ mesons, respectively. The strength of such interactions, not fixed at the saturation density, but free to run, can stiffen or soften the EoS, producing neutron stars whose maximum masses differ by more than one solar mass [49].

As far as the sound velocity in dense matter is concerned, our study corroborates the conclusions reached in [43], namely, models that satisfy correct low density properties and generate maximum star masses around $2 M_{\odot}$, produce squared sound velocities larger than $1 / 3$ still at densities present in the stellar core. However, we verified that when the $\sigma$-cut scheme proposed in Ref. [14] is applied in those RMF models presenting maximum neutron star mass below the range of $1.93 \leqslant M / M_{\odot} \leqslant 2.05$, it makes consistent such a constraint with the sound velocity bound of Ref. [43]. We also found that the isoscalar-vector interaction is an important ingredient in order to validate this consistency.

We have also analysed the possibility of fast cooling induced by the direct Urca process and have checked that 32 out of 34 models could give rise to it.

As a last remark, we point out to the reader two recent RMF parametrizations proposed in [50], namely, BSP and IUFSU*. They were shown to predict maximum stellar masses inside the range of $1.93 \leqslant M / M_{\odot} \leqslant 2.05$ and also to allow the DU process. For the sake of completeness, we have submitted such models to the constraints of [4] and verified that they were approved together with the parameterizations analyzed in this work. We have obtained their central energy densities and the proton fraction related to the threshold of the DU process. The values are $\varepsilon_{c}=6.688 \mathrm{fm}^{-4}$ and $Y_{\mathrm{DU}}=0.139$ for BSP, and $\varepsilon_{c}=6.141 \mathrm{fm}^{-4}$ and $Y_{\mathrm{DU}}=0.138$ for IUFSU* ${ }^{*}$. We also verified that for both parametrizations, the bound $v_{s}^{2}<1 / 3$ is violated at central energy densities smaller than $\varepsilon_{c}$, corroborating once more the findings of [43].

\section{ACKNOWLEDGMENTS}

This work was partially supported by CNPq (grants 300602/2009-0) and FAPESC (Brazil) under project 2716/2012, TR 2012000344. O.L. also acknowledges CNPq, and the support of Grant No. 2013/26258-4 from São Paulo Research Foundation (FAPESP). M.D. acknowledges support from Fundação de Amparo à Pesquisa do Estado do Rio de Janeiro (FAPERJ), Grant No. 111.659/2014.
[1] F.D. Mackie and G. Baym, Nucl. Phys. A 285, 332 (1977).
[2] von Weizscker Zeitschrift fr Physik 96 (78) 431 (1935). 
[3] M. Dutra, O. Lourenço, J. S. Sá Martins, A. Delfino, J. R. Stone, and P. D. Stevenson, Phys. Rev. C 85, 035201 (2012).

[4] M. Dutra, O. Lourenço, S. S. Avancini, B. V. Carlson, A. Delfino, D. P. Menezes, C. Providência, S. Typel, and J. R. Stone, Phys. Rev. C 90, 055203 (2014).

[5] Ermal Rrapaj, Alessandro Roggero, and Jeremy W. Holt, arxiv: 1510.00444.

[6] J. D. Walecka, Ann. Phys. 83, 491 (1974).

[7] J. Boguta and A. R. Bodmer, Nucl. Phys. A 292, 413 (1977).

[8] S. Typel and H. H. Wolter, Nucl. Phys. A 656, 331 (1999).

[9] B. A. Nikolaus, T. Hoch, and D. G. Madland, Phys. Rev. C 46, 1757 (1992); O. Lourenço, M. Dutra, A. Delfino, and R. L. P. G. Amaral, Int. J. Mod. Phys. E, 16, 3037 (2007).

[10] P. B. Demorest, T. Pennucci, S. M. Ransom, M. S. E. Roberts, and J. W. T. Hessels, Nature 467, 1081 (2010).

[11] J. Antoniadis, P. C. C. Freire, N. Wex et al., Science 340, 448 (2013).

[12] S. Weissenborn, D. Chatterjee, J. Schaffner-Bielich, Nucl. Phys. A 881, 62 (2012); S. Weissenborn, D. Chatterjee, J. Schaffner-Bielich, Phys. Rev. C 85, 065802 (2012).

[13] L. L. Lopes and D. P. Menezes, Phys. Rev. C 89, 025805 (2014).

[14] K. A. Maslov, E. E. Kolomeitsev, and D. N. Voskresensky, Phys. Rev. C 92, 052801(R) (2015).

[15] G. Baym, C. Pethick, P. Sutherland, Astrophys. J. 170, 299 (1971).

[16] J. R. Oppenheimer, G. M. Volkoff, Phys. Rev. 33, 374 (1939).

[17] N. K. Glendenning, Compact Stars, Springer, New YorkSecond Edition (2000).

[18] P. Haensel, A. Y. Potekhin, D. G. Yakovlev, Neutron Stars, Equation Of State And Structure, Springer, New York (2006).

[19] B. K. Agrawal, Phys. Rev. C 81, 034323 (2010).

[20] S. K. Dhiman, R. Kumar, and B. K. Agrawal, Phys. Rev. C 76, 045801 (2007).

[21] B.-J. Cai, L.-W. Chen, Phys. Rev. C 85, 024302 (2012).

[22] B. G. Todd-Rutel and J. Piekarewicz, Phys. Rev. Lett. 95, 122501 (2005).

[23] J. Piekarewicz and S. P. Weppner, Nucl. Phys. A 778, 10 (2006).

[24] R. Kumar, B. K. Agrawal, and S. K. Dhiman, Phys. Rev. C 74, 034323 (2006)

[25] A. Sulaksono and T. Mart, Phys. Rev. C 74, 045806 (2006).

[26] F. J. Fattoyev, C. J. Horowitz, J. Piekarewicz, and G. Shen, Phys. Rev. C 82, 055803 (2010).

[27] C. J. Horowitz and J. Piekarewicz, Phys. Rev. C 66, 055803 (2002).

[28] T. Klähn, et al., Phys. Rev. C 74, 035802 (2006).

[29] T. Gaitanos, M. Di Toro, S. Typel, V. Baran, C. Fuchs, V. Greco, and H. H. Wolter, Nucl. Phys. A 732, 24
$(2004)$.

[30] X. Roca-Maza, X. Viñas, M. Centelles, P. Ring, and P. Schuck Phys. Rev. C 84, 054309 (2011).

[31] K. Hebeler, J. M. Lattimer, C. J. Pethick, and A. Schwenk, Phys. Rev. Lett. 105, 161102 (2010).

[32] A. W. Steiner and S. Gandolfi, Phys. Rev. Lett. 108, 081102 (2012); A. W. Steiner, J. M. Lattimer, and E. Brown, Astropys. J. 722, 33 (2010).

[33] J. M. Lattimer and Y. Lim, Astrophys. J. 771, 51 (2013).

[34] J. M. Lattimer and A. W. Steiner, Astrophys. J 784, 123 (2014).

[35] S. Guillot, M. Servillat, N. A. Webb, and R. E. Rutledge, Astrophys. J. 772, 7 (2013).

[36] S. Guillot and R. E. Rutledge, Astrophys. J. 796, L3 (2014).

[37] Rafael Cavagnoli, Debora P Menezes and Constança Providência, Phys. Rev. C 84, 065810 (2011).

[38] S. Gandolfi, J. Carlson, and S. Reddy, Phys. Rev. C 85, 032801(R) (2012).

[39] M. Fortin, J. L. Zdunik, P. Haensel, and M. Bejger, Astron. Astrophys. 576, A68 (2015); arxiv:1408.3052v2.

[40] J. M. Lattimer, C. J. Pethick, M. Prakash, and P. Haensel, Phys. Rev. Lett. 66, 2701 (1991).

[41] V. Dexheimer, R. Negreiros, and S. Schramm, Phys. Rev. C 92, 012801(R) (2015); 91, 055808 (2015); W. G. Newton, K. Murphy, J. Hooker, and B.-A. Li, Astrophys. J. 779, L4 (2013).

[42] P. M. Hohler and M. A. Stephanov, Phys. Rev. D 80, 066002 (2009); J. Noronha-Hostler, J. Noronha, and C. Greiner, Phys. Rev. Lett. 103, 172302 (2009); N. Chamel et al., Astron. Astrophys. 553, A22 (2013).

[43] Paulo Bedaque and Andrew W. Steiner, Phys. Rev. Lett. 114, 031103 (2015).

[44] M. G. Paoli and D.P. Menezes, Eur. Phys. J A 46, 413 (2010).

[45] N. Glendenning, Phys. Lett. B 114, 392 (1982).

[46] K. A. Maslov, E. E. Kolomeitsev, D. N. Voskresensky, Phys. Lett. B 748, 369 (2015); I. Bednarek, P. Haensel, J. L. Zdunik, M. Bejger, and R. Mańka, Astron. Astrophys. 543, A157 (2012); M. Oertel, C. Providência, F. Gulminelli, and A. R. Raduta, J. Phys. G 42, 075202 (2015); Phys. Part. Nucl. 46, 830 (2015); A. Sulaksono and B. K. Agrawal, Nucl. Phys. A 895, 44 (2012); R. Mallick, Phys. Rev. C 87, 025804 (2013); N. Gupta and P. Arumugam, Phys. Rev. C 88, 015803 (2013); D. Lonardoni, A. Lovato, S. Gandolfi, and F. Pederiva, Phys. Rev. Lett. 114, 092301 (2015); I. Bednarek, Phys. Part. Nucl. 46, 816 (2015).

[47] J. R. Torres, F. Gulminelli, and D. P. Menezes, arxiv: $1510.01381 \mathrm{v} 2$.

[48] S. A. Moszkowski. Phys. Rev. D 9, 1613 (1974).

[49] H. Müller and B. D. Serot, Nucl. Phys. A 606, 508 (1996).

[50] B. K. Agrawal, A. Sulaksono, and P.-G. Reinhard, Nucl. Phys. A 882, 1 (2012). 